\title{
Ruthenium-Catalyzed Transfer Hydrogenation of Alkynes by Tributylamine
}

\author{
Chan Sik Cho, ${ }^{\star}$ Dong Tak Kim, and Sang Chul Shim \\ Department of Applied Chemistry, Kytmgpook Vational Lniversity, Daegu 702-701, Korea. 'E-mail: cschoathntackr \\ Received Mav 21, 2009. Accepted August 2, 2009
}

Key Words: Alkenes. Alkynes. Ruthenium catalyst. Transfer hỵdrogenation. Tributylamine

In contrast to conventional reduction methods, which frequently require a high hydrogen pressure and hazardous reducing agents. transition metal-catalyzed transfer hỵdrogenation has some unique advantages in its simplicity and avoidance of cumbersome reducing agents. ${ }^{1.2}$ Many hydrogen donors combined with a variety of transition metal catalysts so far have been developed for such a transfer hy'drogenation. ${ }^{1}$ Among them. in connection with this report. several transition metals revealed a catalytic activity to the transfer of hydrogen from trialky lamines. ${ }^{3}$ For example. Tsuji et al. have reported that aldehydes react with allylic acetates in the presence of a nithenium catalyst along with triethylamine to give homoallylic alcohols and the hydrogen source for the products is triethylamine. ${ }^{3 e}$ It is also reported that dipheny lacetylene is used as a sacrificial hydrogen acceptor in a ruthenium-catalyzed șynthesis of esters from aldelydes and alcohols. ${ }^{-1}$ During the course of our ongoing studies on ruthenium catalysis. we recently found an unusual type of transfer hydrogenation between ketones (or secondary alcohols) and primary alcohols (or aldehydes) accompanied by carbon-carbon bond formation under a ruthenium-catalyzed redox shuttle. ${ }^{5.8}$ Under these circumstances. herein we report a nithenium-catalyzed transfer hydrogenation of alkynes to trans-alkenes by a trialkylamine (Scheme 1).

The results of several attempted transfer hydrogenation of dipheny lacety lene (1a) under various conditions are listed in Table 1 . Treatment of 1 a in dioxane in the presence of a catalytic amount of $\mathrm{RuCl}_{2}\left(\mathrm{PPh}_{3}\right)_{3}$ along with $\mathrm{Bu}_{3} \mathrm{~N}$ afforded transstilbene (2a) in $87 \%$ yield with concomitant formation of cis-stilbene (3) (2\% yield) with complete conversion of 1 a (nu l). Performing the reaction for a longer reaction time ( $15 \mathrm{~h}$ ) gave $2 \mathbf{a}$ as the sole reduction product (run 2). The amount of $\mathrm{RuCl}_{3}\left(\mathrm{PPh}_{3}\right)_{3}$ affected the distribution of reduced products. a smaller amount of $\mathrm{RuCl}_{3}\left(\mathrm{PPh}_{3}\right)_{3}$ even under a larger amount of $\mathrm{Bu}_{3} \mathrm{~N}$ resulting in preferential formation of 3 (run 3). This result clearly indicates that $\mathrm{RuCl}_{3}\left(\mathrm{PPh}_{3}\right)_{3}$ play's a role for the selectivity of 2 a to 3 . Lower reaction temperature resulted in lower yield and selectivity of reduced products (nın 4). The reaction proceeds even in the absence of $\mathrm{Bu}_{3} \mathrm{~N}$. however, the yield and selectivity of products were lower than those when the reaction was carried out in the presence of $\mathrm{Bu}_{3} \mathrm{~N}$ (run 5 ). The reduction seems to be due to transfer hydrogenation from

$$
\mathrm{R}^{1}=\mathrm{R}^{2} \stackrel{[\mathrm{Ru}], \mathrm{Bu}_{3} \mathrm{~N}}{\longrightarrow}[\mathrm{R}^{1}\left[\mathrm{R}^{2}\right] \stackrel{[\mathrm{Ru}]}{\longrightarrow} \underset{\mathrm{R}^{1}}{\rightleftharpoons} \overbrace{}^{\mathrm{R}^{2}}
$$

dioxane to 1a. It is known that dioxane has been used as a hydrogen donor in transition metal-catalyzzed transfer hỵdrogenation. ${ }^{101}$ From the activity of several ruthenium precursors examined. $\mathrm{RuCl}_{2}\left(\mathrm{PPh}_{3}\right)_{3}$ is revealed to be the catalyst of choice and other catalyst precursors such as $\mathrm{RuH}_{2}\left(\mathrm{PPh}_{2}\right)_{4}, \mathrm{RuCl}_{2}$ $(=\mathrm{CHPh})\left(\mathrm{PCy}_{3}\right)_{2}, \mathrm{RuCl}_{5}, n \mathrm{H}_{2} \mathrm{O} / 3 \mathrm{PPl}_{3}$ and $\mathrm{Ru}_{3}(\mathrm{CO})_{12}$ resulted in either lower yield or lower selectivity of products (runs 6-9).

After the reaction conditions have been established. a series of diarylalkynes 1 were employed to investigate the reaction scope and several representative results are summarized in Table 2. " With diarylalky nes (1a-g) having electron donating and withdrawing substituents. the reductive isomerized diaryl alkenes $(2 \mathrm{a}-\mathrm{g})$ were formed in the range of $60-86 \%$ yields. Here again, cis-alkenes were scarcely produced. The product yield was not significantly affected by the position of the substituent on the aromatic ring. whereas the electronic nature of that had some relevance to the product yield. 2-(Phenylethynyl) naphthalene (1h) was also reductively isomerized under the employed conditions to give (E)-2-sty yylnaphthalene (2h) in $74 \%$ y ield. With diaryl alky ne $1 \mathrm{i}$, which has heteroaryl group. the corresponding trans-alkene $2 \mathbf{i}$ was also produced and the product yield was lower than that when compared to the reaction with previously described diaryl alkynes (1a-h). The reaction proceeds likewise with diaṇl alkỵnes (1jand 1k) having electron donating and withdrawing substituents at both aromatic rings

Table 1. Reductions under several conditions ${ }^{a}$

\begin{tabular}{|c|c|c|c|c|c|}
\hline \multicolumn{2}{|c|}{$\begin{array}{c}\mathrm{Ph}=\mathrm{Ph} \\
1 \mathrm{a}\end{array}$} & $\frac{[R u]}{\text { dioxane }}$ & $\mathrm{Ph}=$ & + & 3 \\
\hline \multirow{2}{*}{ Run } & \multirow{2}{*}{\multicolumn{2}{|c|}{ Ruthenium catalyst }} & Conv. ${ }^{2}(\%)$ & \multicolumn{2}{|c|}{ Yield $^{b}(\%)$} \\
\hline & & & of $1 \mathbf{a}$ & $2 \mathbf{a}$ & 3 \\
\hline 1 & \multicolumn{2}{|c|}{$\mathrm{RuCl}_{2}\left(\mathrm{PPh}_{3}\right)_{3}$} & 100 & 87 & 2 \\
\hline $2^{c}$ & \multicolumn{2}{|c|}{$\mathrm{RuCl}_{2}\left(\mathrm{PPh}_{3}\right)_{3}$} & 100 & 89 & trace \\
\hline $3^{d}$ & \multicolumn{2}{|c|}{$\mathrm{RuCl}_{2}\left(\mathrm{PPh}_{3}\right)_{3}$} & 100 & 16 & 48 \\
\hline $4^{e}$ & \multicolumn{2}{|c|}{$\mathrm{RuCl}_{2}\left(\mathrm{PPh}_{3}\right)_{3}$} & 42 & 7 & 19 \\
\hline$\xi^{\prime}$ & \multicolumn{2}{|c|}{$\mathrm{RuCl}_{2}\left(\mathrm{PPh}_{3}\right)_{3}$} & 30 & 7 & 18 \\
\hline 6 & \multicolumn{2}{|c|}{$\mathrm{RuH}_{2}\left(\mathrm{PPh}_{3}\right)_{4}$} & 81 & 6 & 52 \\
\hline 7 & \multicolumn{2}{|c|}{$\mathrm{RuCl}_{2}(=\mathrm{CHPh})\left(\mathrm{PCy}_{3}\right)_{2}$} & 100 & 57 & 26 \\
\hline 8 & \multicolumn{2}{|c|}{$\mathrm{RuCl}_{3} \cdot n \mathrm{H}_{2} \mathrm{O} / 3 \mathrm{PPh}_{3}$} & 100 & 81 & 12 \\
\hline 9 & \multicolumn{2}{|c|}{$\mathrm{Rul}_{3}(\mathrm{CO})_{12}$} & 100 & 54 & 4 \\
\hline
\end{tabular}

"Reaction conditions: 1 a $(0.5 \mathrm{mmol})$, ruthenium catalyst $(0.025 \mathrm{mmol})$. $\mathrm{Bu}_{3} \mathrm{~N}(0.2 \mathrm{mmol})$. dioxane $(5 \mathrm{~mL}) .180^{\circ} \mathrm{C}$, for $5 \mathrm{l}$. under Ar "Determined by GLC. ${ }^{\circ} \mathrm{For} 15$ h. ${ }^{d} \mathrm{RuCl}_{2}\left(\mathrm{PPl}_{13}\right)_{3}(0.005$ mmol $) .{ }^{e}$ At $120^{\circ} \mathrm{C}$. In the absence of $\mathrm{Bu}{ }_{3} \mathrm{~N}$. 
Table 2. Reduction of alkynes 1 to alkenes 2 under $\mathrm{RuCl}_{-}\left(\mathrm{PPl}_{3}\right)_{3} /$ $\mathrm{Bu} \mathrm{N}^{4 i}$

\begin{tabular}{|c|c|c|c|}
\hline \multicolumn{2}{|c|}{$\mathrm{R}^{1}=\mathrm{R}^{2}$} & $\frac{\mathrm{l}_{2}\left(\mathrm{PPh}_{3}\right)_{3}, \mathrm{Bu}_{3} \mathrm{~N}}{\text { ne, } 180^{\circ} \mathrm{C}, 15 \mathrm{~h}}$ & \multirow[t]{2}{*}{$\underbrace{R^{1}}_{2}$} \\
\hline & 1 & & \\
\hline & \multicolumn{2}{|c|}{ Alkynes 1} & Isolated vield \\
\hline & $\mathrm{R}^{1}=$ & $\mathrm{R}^{2}=$ & $(\%)$ \\
\hline $1 \mathbf{a}$ & $\mathrm{Ph}$ & $\mathrm{Ph}$ & 83 \\
\hline $1 b$ & $\mathrm{Ph}$ & $2-\mathrm{CH}_{3} \mathrm{C}_{6} \mathrm{H}_{4}$ & 84 \\
\hline $1 c$ & $\mathrm{Pl}$ & $3-\mathrm{CH}_{3} \mathrm{C}_{6} \mathrm{H}_{4}$ & 84 \\
\hline Id & $\mathrm{Ph}$ & $4-\mathrm{CH}_{3} \mathrm{C}_{6} \mathrm{H}_{4}$ & 86 \\
\hline le & $\mathrm{Ph}$ & $4-\mathrm{CH}_{3} \mathrm{OC}_{6} \mathrm{H}_{4}$ & 60 \\
\hline If & $\mathrm{Ph}$ & $2-\mathrm{FC}_{6} \mathrm{H}_{4}$ & 78 \\
\hline $1 g$ & Pli & $4-\mathrm{FC}_{6} \mathrm{H}_{+}$ & 70 \\
\hline $1 \mathrm{~h}$ & Ph & 2-Naphthyl & 74 \\
\hline $1 \mathrm{i}$ & $\mathrm{Ph}$ & 3-Pyindyl & 63 \\
\hline $1 \mathbf{j}$ & $4-\mathrm{CH}_{2} \mathrm{C}_{6} \mathrm{H}_{4}$ & $4-\mathrm{CH}_{3} \mathrm{OC}_{6} \mathrm{H}_{4}$ & 89 \\
\hline $1 \mathrm{k}$ & $4-\mathrm{CH}_{2} \mathrm{C}_{6} \mathrm{H}_{4}$ & $4-\mathrm{FC}_{6} \mathrm{H}_{4}$ & 72 \\
\hline 11 & $4-\mathrm{CH}_{3} \mathrm{OC}_{6} \mathrm{H}_{4}$ & Hexyl & $42^{b}$ \\
\hline
\end{tabular}

${ }^{a}$ Reaction conditions: $1(0.5 \mathrm{mmol})$. ruthenium catalyst $(0.025 \mathrm{mmol})$. $\mathrm{Bu} \mathrm{u}_{3} \mathrm{~N}(0.2 \mathrm{~mm} \mathrm{~m})$, dioxane $(5 \mathrm{~mL}) .180^{\circ} \mathrm{C}$. for $15 \mathrm{~h}$. under Ar. ${ }^{\circ}$ Deternined by ${ }^{\mathrm{l}} \mathrm{H}$ NMR.

to give the corresponding diaryl alkenes ( $2 \mathbf{j}$ and $2 \mathbf{k}$ ) in similar yields. On the other hand. although the reaction proceeds with alkyl aryl alky'ne 11. the isolation of pure trans alkene product from crude mixture met with failure since the product is exactly eclipsed with a small amount of several unidentifiable compounds on chromatographies.

As to the reaction pathway. although it is not yet fully understood this seems to proceed $v a$ an initial transfer hydrogenation of alkyne by tributylamine as well as dioxane to form cis-alkene, which in turn triggers isomerization to give trans-alkene. We confirmed in a separate experiment that the yield of 2a increased from $9 \%(\mathrm{~h}) .17 \%(2 \mathrm{~h}) .24 \%(3 \mathrm{~h}) .72 \%(4 \mathrm{~h})$. to $87 \%(5 \mathrm{~h})$. while that of 3 decreased from $45 \%(1 h) .46 \%(2 h) .57 \%(3 h)$. $10 \%(+h)$. to $2 \%(5 \mathrm{~h})$. Furthermore. we also confirmed that cis-stilbene was completely isomerized to trans-stilbene under $\mathrm{RuCl}_{-}\left(\mathrm{PPh}_{3}\right)_{3}(5 \mathrm{~mol} \%) /$ dioxane $/ 180{ }^{\circ} \mathrm{C} / 5$ h. These additional experiments along with the result of nun 3 of Table 1 clearly show that 3 is initially formed by ruthenium-catalyzed transfer hydrogenation followed by ruthenium-catalyzed isomerization to 2a It is known that cis-alkenes are isomerized to trans-alkenes via a carbocation in the presence of a palladium catalyst. ${ }^{12.13}$

In summary. it has been shown that a variety of diaryl alkynes are converted into trans-alkenes under $\mathrm{RuCl}_{2}\left(\mathrm{PPh}_{3}\right)_{3} / \mathrm{Bu}_{3} \mathrm{~N}$ via initial transfer hydrogenation from $\mathrm{Bu}_{j} \mathrm{~N}$ to diaryl alkynes to form cis-alkenes and subsequent ruthenium-catalyzed isomerization.

Acknowledgments. This work was supported by the Korea Research Foundation Grant funded by the Korean Government (MOEHRD. Basic Research Promotion Fund) (KRF2008-331-C00176).

\section{References}

1. For reviews on transition metal-catalyzed transfer hydrogenation, see (a) Noyori, R:; Hashiguchi, S. Acc Chem. Res. 1997. 30, 97. (b) Naota, T: Talkaya, H.; Murahashi, S.-I. Chem. Rev. 1998. 98, 2599. (c) Palmer, M.; Wills, M. Tetrahedron: Asmmetry 1999. 10, 2045

2. (a) In Compiehensive Organometallic Chemistiv; Trost, B. M.; Fleming, I., Eds.: Pergamon: Oxford, 1991: Vol. 8. (b) Hudlicky, M Reductions in Oiganic Chemistry. American Chemical Society: Washington, D. C., 1996.

3. (a) Murahashi, S.-I.; Hirano, T: Yano, T. J. Am. Chent Soc. 1978, 100,348 (b) Murahashi, S.-I.; Yano, T. J. Am. Chem. Soc. 1980, 102, 2456. (c) Murahashi, S.-I.; Yoshimura, N.: Tsumiyama, T.; Kojima, T. $J$. Am. Chem. Soc. 1983, 105, 5002 (d) Wilson, R. B.: Laine, R. M. J. Am. Chemt. Soc. 1985, 107, 361. (e) Tsuji, Y; Mukai, T.: Kondo, T.: Watanabe, Y. J. Organomet. Chem. 1989. $369 . \mathrm{C} 51$

4. (a) Blum, Y.; Reshef, D.; Shvo, Y. Tetwhedron Lett. 1981, 22 , 1541. (b) Murahashi, S.-I.; Ito, K.; Naota, T.; Maeda, Y. Tetrohedron Lett. 1981, 22, 5327. (c) Murahashi, S.-I.; Naota, T.; Ito, K:; Maleda, Y; Taki, H. J. Oig. Chem. 1987, 52,4319

5. (a) Cho, C. S.: Kim, B. T.: Kim, T.-J.: Shim, S. C. J. Org Chem. 2001, 66, 9020. (b) Cho, C. S.; Kim, B. T.; Kim, T.-J.; Shim, S. C. Tetrohedron Letr. 2002, 43, 7987. (c) Cho, C. S.; Kim, B. T: Kim. H.-S.: Kim. T.-J.: Shim. S. C. Oiganometallics 2003, 22. 3608. (d) Cho, C. S. J. Mol. Cat. A. Chem. 2005, 240, 55 . (e) Cho, C. S.; Shim, S. C.J. Organomet. Chent 2006, 691, 4329. (t) Cho, C. S. J. Mol. Cat . A: Chent 2007, 267, 49.

6. Several other transition metal precursors have also been used for such couplings: (a) Taguchi, K.: Nakagawa, H.: Hirabavashi, T: Sakaguchi, S.; Ishii, Y. J. Am. Chem. Soc. 2004, 126, 72. (b) Fujita, K.; Asai, C.; Yanaguchi, T.; Hanasaka, F.; Yamaguchi, R. Ong. Leth. 2005, 7, 4017. (c) Onodera, G.; Nishibavashi, Y.; Uemura, S. Angew: Chem Int Ed 2006, 45, 3819. (d) Slatford, P. A.; Whittlesey, M. K.: Willians, I. M. K. Tetrahedron Lett. 2006. 47,6787 .

7. These reactions could also be applied to transition metalcatalyzed friedlander quinoline synthesis by oxidative cyclization of 2-aminobenzyl alcohol with ketones and secondary alcohols: Cho, C. S.; Ren, W. X: Yoon, N. S. J. Hol Cat . A: Chem. 2009, 299, 117 and references cited therein.

8. Guillena, G.: Ramón, D. J:; Yus, M. Angew: Chem. Int. Ed. 2007. $\$ 6.2358$.

9. For our reports using trialliy lamines as an alkylating agent: (a) Cho, C. S.; Oh, B. H.; Kim, J. S.; Kim, T.-J.; Shim, S. C. Chem. Conmm 2000, 1885. (b) Cho, C. S.; Kim, B. T.; Lee, M. T.; Kim, T.-T.; Shim, S. C. Angew. Chem. Int Ed 2001, 40,958 . (c) Cho, C. S.; Kim, T. K; Kim, B. T; Kim, T.-J.: Shim, S. C. J. Organontet. Chem. 2002, 650, 65 .

10. (a) Nishiguchi, T.; Tachi, K. Fukuzumi, K. $J$. Am. Chent Soc: 1972, 94,8916. (b) Nishiguchi, T: Fukuzumi, K. J. Am. Chem. Soc. 1974, 96, 1893 (c) Imai, H.: Nishiguchi, T.; Fukuzumi, K. J. Org. Chemt $1976,41,665$.

11. General experimental procedure: To a $50 \mathrm{~mL}$ pressure vessel were added alkyne $\left(0.5 \mathrm{mmol}^{2}\right), \mathrm{RuCl}_{2}\left(\mathrm{PPh}_{3}\right)_{3}(0.025 \mathrm{mmol})$, $\mathrm{Bu} l_{3} \mathrm{~N}(0.2 \mathrm{mmol})$ and dioxane $(5 \mathrm{~mL})$. After the system was flushed with argon, the reaction mixture was allowed to react at $180^{\circ} \mathrm{C}$ for $15 \mathrm{~h}$. The reaction mixture was passed through a short silica gel columu (ethyl acetate-hexane) to eliminate a ruthenium. Removal of the solvent left a crude misture, which was separated by thin layer chromatography (silica gel, ethyl acetate-hesane mixture) to give trons-alkenes.

12. Yu, T.; Gaunt, M. J.; Spencer, T. B. J. Org. Chent. 2002,67, 4627.

13. Sen. A.: Lai, T.-W. Horg. Chem 1981, 20,4036. 